\section{Eigenständig und integriert}

\section{Die Umweltkostenrechnung ist als wesentlicher Bestandteil des Umweltmanage- ments ein oft noch vernachlässigtes Gebiet. Praxistauglichkeit und eine Ausge- staltung im Sinne eines aktiven und ingetrierten Umweltschutzes sind nur in Ausnahmefällen gegeben, wie ein Gutachten von IÖW und IÖB zeigt. Wie hat sich die Umweltkostenrechnung entwickelt und wie kann sie verbessert werden?}

Von Klaus Fichter m Auftrag des hessischen Wirtschaftsministeriums hat das Institut für Ökologische Wirtschaftsforschung (IÖW) in Zusammenarbeit mit dem Institut für Ökologische Betriebswirtschaft (IÖB, Prof. Eberhard Seidel, Siegen) ein Gutachten zur betrieblichen Umweltkostenrechnung erstellt (1). Dieses gibt einen systematischen Überblick über die in den vergangenen 20 Jahren in Theorie und Praxis entwickelten Ansätze der betrieblichen Umweltkostenrechnung und bewertet ihr Entwicklungspotential mit Blick auf einen immer wichtiger werdenden aktiven und integrierten Umweltschutz.

Seit Mitte der 70er Jahre läßt sich eine zunehmende Auseinandersetzung mit Fragen der betrieblichen Umweltkostenrechnung beobachten. Die Konzepte und Berechnungen in den 70er und 80er Jahren beschränkten sich auf die Ermittlung der Kosten von nachsorgenden Umweltschutzanlagen und -maßnahmen und wurden als solche gesondert durchgeführt. Seit Ende der 80er Jahre zielen die Konzepte der Umweltkostenrechnung auf eine Erweiterung und umfassende Nutzung bestehender Kostenrechnungssyteme ab. Dafür lassen sich folgende Gründe nennen:

Zum einen sind seit den 70er Jahren die betrieblichen Umweltschutzkosten kontinuierlich angestiegen. Zum anderen hat die Unternehmenspraxis gezeigt, daß mit Umweltschutzmaßnahmen auch Kosten gesenkt werden können. Und der steigende Wettbewerbs- und Kostendruck in den 90er Jahren am Standort Deutschland hat die Suche nach Kostensenkungspotentialen ausgelöst.

Die bislang entwickelten Ansätze für eine betriebliche Umweltkostenrechnung lassen sich, wie in der Tabelle dargestellt, nach zwei grundlegenden Kriterien differenzieren :

- nach den im Mittelpunkt stehenden Kosten (Umweltschutzkosten, stoff- und energieflußbezogene Kosten, externe Kosten usw.)
- und nach dem zugrundegelegten Kostenrechnungssystem (Sonderrechnungen, Voll-, Teil-, Prozeßkostenrechnung etc.)

\section{Bislang fehlt die Integration}

In der Unternehmenspraxis existieren derartig verknüpfte Rechnungssysteme, die über die Kostenwirkung einzelner Umweltschutzanlagen oder -maßnahmen hinausgehen, erst seit 1992/1993 (2-5). Die weitreichendsten Ansätze sind bislang, wie die Untersuchung zeigte, bei den Firmen Kunert (D), Amoco Oil (USA) und Ontario Hydro (Kanada) zu finden. Aber auch dort haben sie im wesentlichen noch Projektcharakter, das heißt eine vollständige Integration der Umweltkostenrechnung in das laufende Kostenrechnungssystem und eine Integration des Umweltkostenmanagements in das gesamte Mangementsystem stehen auch hier noch aus. Ein häufig genannter Grund für die Scheu vor der Anwendung erweiterter Systeme sind Zweifel an der Praktikabilität. Soll die Umweltkostenrechnung praxistauglich sein und den Anforderungen eines aktiven und integrierten Umweltschutzes gerecht werden, so muß diese:

- Synergieeffekte bei der Verfolgung ökologischer und ökonomischer Ziele systematisch hervorbringen,

- konkrete Entscheidungshilfen für Mitarbeiter und Geschäftsführung liefern,

- auf Kostenbegriffe abheben, die eine eindeutige Abgrenzung verschiedener Kostenarten ermöglichen,

- einen möglichst geringen Aufwand bei der Erfassung sicherstellen,

- konkrete Ansatzpunkte für die Entwicklung kostensenkender Maßnahmen bieten,

- die Kostenwirkung von Umweltschutzmaßnahmen ausreichend genau und umfassend beschreiben und

- Kosten des unterlassenen Umweltschutzes transparent machen.

\section{Die richtige Wahl}

Mit Blick auf die Aufgaben einer Umweltkostenrechnung läßt sich feststellen, daß sich die Anforderungen nicht wesentlich von der Verrechnung anderer Kosten unterscheidet. Im Mittelpunkt steht die verursachungsgerechte Zuordnung von Gemeinkosten und Fixkosten auf einzelne Kostenstellen und Kostenträger und
Ansüłze der Umweltkostenrechnung im Überblick

\begin{tabular}{|c|c|c|c|c|}
\hline . & $\begin{array}{l}\text { Umweltschutz- } \\
\text { kosten }\end{array}$ & $\begin{array}{l}\text { Stoff- und energie- } \\
\text { flußbezogene } \\
\text { Kosten }\end{array}$ & $\begin{array}{l}\text { Potentielle } \\
\text { Umwelt(schutz)- } \\
\text { kosten }\end{array}$ & $\begin{array}{l}\text { Externe } \\
\text { Umweltkosten }\end{array}$ \\
\hline $\begin{array}{l}\text { Sonderrechnungen/ } \\
\text { Auswertungen }\end{array}$ & $\begin{array}{l}\text { Kosten der Emissions- } \\
\text { minderung }\end{array}$ & & $\begin{array}{l}\text { Umweltbudget- } \\
\text { rechnung }\end{array}$ & $\begin{array}{l}\text { Costs of Environ- } \\
\text { mental Effects }\end{array}$ \\
\hline Vollkostenrechnung & $\begin{array}{l}\text { Umweltschutzkosten } \\
\text { auf Vollkostenbasis }\end{array}$ & $\begin{array}{l}\text { Reststoffkostenrech- } \\
\text { nung }\end{array}$ & $\begin{array}{l}\text { Berücksichtigung } \\
\text { von Wagniskosten }\end{array}$ & Full Cost Accounting \\
\hline Teilkostenrechnung & $\begin{array}{l}\text { Umweltschutzorien- } \\
\text { tierte Kostenrechnung } \\
\text { Mehrstufige Deckungs- } \\
\text { beitragrechnung }\end{array}$ & & $\begin{array}{l}\text { Ökologieorientierte } \\
\text { Kostenrechnung }\end{array}$ & \\
\hline $\begin{array}{l}\text { Prozeßkostenrech- } \\
\text { nung }\end{array}$ & $\begin{array}{l}\text { Activity Based } \\
\text { Accounting }\end{array}$ & $\begin{array}{l}\text { Stoff- und energie- } \\
\text { flußorientierte } \\
\text { Kostenrechnung }\end{array}$ & & \\
\hline
\end{tabular}

Bislang nicht in der Unternehmenspraxis umgesetzte Ansätze

Quelle: Unverôffentlichtes IÖW-Gutachten im Auftrog des hessischen Wirtschaftsministeriums. Betriebliche Umweltkostenrechnung - Stand, Anforderungen und Entwicklungsperspektiven. Erstellt von K. Fichter, T. Loew, E. Seidel und R. Antes, Berlin 1996. Dos Gutachten erscheint im Frühjohr 1997 beim SpringerVerlag. 


\section{Ontario Hydro}

Anfang der 90 er Jahre begann das kanadische Energieversorgungsunternehmen Ontario Hydro im Rohmen des Konzeptes des Full Cost Accounting (FCA) mit ausführlicheren Arbeiten zur Quantifizierung und Monetarisierung der vom Unternehmen ausgehenden Umweltwirkungen (5). Beim FCA handelt es sich um einen umfassenden Versuch der Erfassung der vom Unternehmen ausgehenden Umweltwirkungen sowie der damit verbundenen internen und externen Kosten. Im Gegensatz zu anderen Ansötzen der Umweltkostenrechnung wird damit die Dimension der externen Effekte und Kosten einbezogen. Das FCA wird dabei nicht als isoliertes Entscheidungsinstrument gesehen, sondern als eines von mehreren Instrumenten, das umweltbezogene Informationen und Bewertungen für Entscheidungsprozesse liefert. Allerdings darf trotz der guten Absicht, ouch externe Kosten in die Entscheidungsfindung einfließ̉en zu lassen nicht übersehen werden, daß die Monetarisierung externer Iffekte methodisch äußerst schwierig (z.B. Summations- und Distanzschöden) und deshalb nach wie vor sehr umstritten. Insbesondere bei der Erfassung der externen Kosten der Kernenergie und der globalen Folgekosten der Energieerzeugung auf Bosis fossiler Brennstoffe (Treibhauseffekt) sind hier grobe Fragezeichen zu setzen.

die Abschätzung der Kostenwirkung einzelner Maßnahmen beziehungweise unterlassener (Umweltschutz-) Maßnahmen.

Einzelne Systeme unterscheiden sich in ihrer Fähigkeit zur verursachungsgerechten Zuordnung, insbesondere von fixen Gemeinkosten, deutlich. So zeigt beispielsweise die Prozeßkostenrechnung Vorteile gegenüber der Direktkostenrechnung oder ,„älteren“ Formen der Vollkostenrechnung.

Für die praktische Umsetzung ergibt sich aus dieser Tatsache jedoch ein Dilemma. Der „objektiven Tauglichkeit“ eines Kostenrechnungssystems steht die ,subjektive Attraktivität“, also die Erwünschtheit aus Sicht der Unternehmenspraxis, gegenüber. Die praktische Implementierung und dauerhafte Verankerung einer Umweltkostenrechnung kann nur unter Berücksichtigung des Standes der Kostenrechnung in der Praxis erfolgen. In den letzten Jahren hat sich die Deckungsbeitragsrechnung in Unternehmen aller Größenklassen immer mehr durchgesetzt und damit eine große Akzeptanz erfahren. Dabei stand und steht in der Regel das Produkt im Mittelpunkt dieser Rechnung.

Auf kurze Sicht hat also die Anpassung der Umweltkostenrechnung an die derzeit vorhandenen Kostenrechnungssysteme Vorrang. Deshalb müssen die Konzepte der Umweltkostenrechnung flexibel an bestehende Systeme ankopperlbar sein. Langfristig wäre es aber im Sinne einer effizienten Umweltkostenrechnung wünschenswert, wenn die Unternehmen die umfassende Prozeßkostenrechnung einführen würden.

\section{Flußkostenrechnung - Ansatz mit Zukunft}

Unter den zwölf im Rahmen des Gutachtes analysierten Konzepten der Umweltkostenrechnung wird diesen Anforderungen die stoff- und energieflußorientierte Kostenrechnung (Flußkostenrechnung) und die Reststoffkostenrechnung (als eine Form der Flußkostenrechnung) am ehesten gerecht. Der zentrale Vorteil der Flußkostenrechnung besteht darin, daß diese an den betrieblichen Stoff- und Energieströmen ansetzt und von der einfachen aber plausiblen Annahme ausgeht, daß mit der Vermeidung oder Verminderung von Material- und Reststoffströmen eine geringere (produktionsbedingte) Umweltbelastung einhergeht. Die Flußkostenrechnung muß sich daher im weiteren nicht mit der Frage beschäftigen, welche betrieblichen Maßnahmen zu welchem Anteil dem Umweltschutz dienen. Während sich bisherige Ansätze vorrangig mit der Identifizierung und Separierung von Umweltschutzkosten beschäftigen, stehen bei der Fluß- und der Reststoffkostenrechnung die verursachungsgerechtere Zuordnung jeglicher Kosten auf bestimmte Stoffe, Energien oder reststoffverursachende Aktivitäten im Mittelpunkt. Dies wird mittels der erfaßten Stoff- und Energieströme möglich.

Mit der Flußkostenrechnung werden mehr Synergien zwischen ökonomischen und ökologischen Zielen freigesetzt und die bislang übliche Gleichung ,mehr Umweltschutz gleich mehr Kosten “ widerlegt. Denn es werden Kostentreiber und -senkungsmöglichkeiten ermittelt, die im Einflußbereich des Unternehmens liegen. Diese Rechnungsart ist daher entscheidungsorientiert.

\section{Einbettung in das Managementsystem}

Viele der bislang entwickelten Ansätze der Umweltkostenrechnung beschränken sich auf Vorschläge zur Ermittlung bestimmter Umweltkostenarten oder auf Rechnungssysteme mit Hilfe derer betriebliche Umweltkosten auf einzelne Kostenstellen oder -träger verrechnet werden sollen. Gerade bei den bislang nicht praktizierten Ansätzen bleiben dabei drei zentrale Fragen zur Umweltkostenrechung offen:

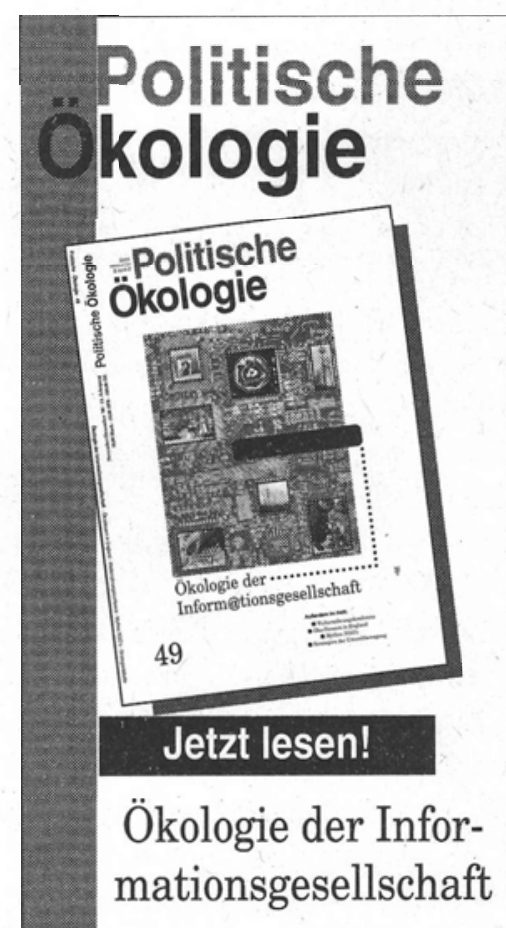

$D_{\text {verspricht weiteres }}^{\text {as Informationszeitalter }}$ Wohlstandwachstum bei gleichzeitiger Entlastung der Umwelt. Doch wer im Netz surft hinterläßt nicht nur virtuelle Spuren. Entlasten Telearbeit, Teleshopping oder virtuelles Reisen tatsächlich die Umwelt?

Verringern elektronische Verkehrsleitsysteme den Fahrzeugstrom? Verschärfen nicht vielmehr Millionen ausgedienter Computer das Müllproblem? Und was ist mit der Gesundheitsgefährdung durch Elektrosmog?

F.-O. Wolf/K. Dräger: Die Verheißungen der Informationsgesellschaft · Interview mit S. Mosdorf über Ökologie und Informationsgesellschaft · V. Schütte: Umweltrelevanz von Telearbeit · H. Klewe: Telematik und Verkehr .

C. Duncker: Virtuelles Reisen . O. Tödter/A. Rolf: Teleshopping Einkauf mit Nebenwirkungen . J. Malley: Multi-Media und Nachhaltigkeit · R. Grieshammer: Perspektiven der Informationsgesellschaft - F. Kochem: Gesundheitsrisiken durch Elektrosmog

Jetzt für DM 19,80 • ökom GmbH überall in der - Waltherstr. 29, Rgb. Bahnhofspresse 80337 München erhältlich oder direkt • Tel.: 089/544 184-0 bestellen bei: - Fax: 089/544 184-99 
- Für welche praxisrelevanten Aufgabestellungen und Entscheidungssituationen soll sie Informationen liefern?

- In welchem Verhältnis steht sie zu anderen betrieblichen Informationssystemen (Betriebsökobilanzen, Qualitätssicherung usw.), das heißt welche entscheidungsrelevanten Informationen kann sie liefern und welche müssen oder sollen durch andere Informationssysteme geliefert werden?

- Wie kann sie in den Management- und Controllingprozeß eines Unternehmens integriert werden?

Diese Kritik gilt für alle Ansätze mit Ausnahme der Fluß- und Reststoffkostenrechnung, dem ActivityBased Accounting (bei Amoco Oil) und dem Full Cost Accounting (bei Ontario Hydro).

Ansätze der Umweltkostenrechnung, die als isoliertes Controllinginstrument konzipiert werden, unterliegen einer doppelten Gefahr: Erstens, vernachlässigen sie betriebliche Entscheidungsrealitäten wie ökologischen Wirkungen, Kundenanforderungen, gesetzlichen Vorschriften, technischer Machbarkeit, Qualitätsanforderungen etc. Zweitens überfordern sie die Kostenrechnung als Informationsquelle und las-

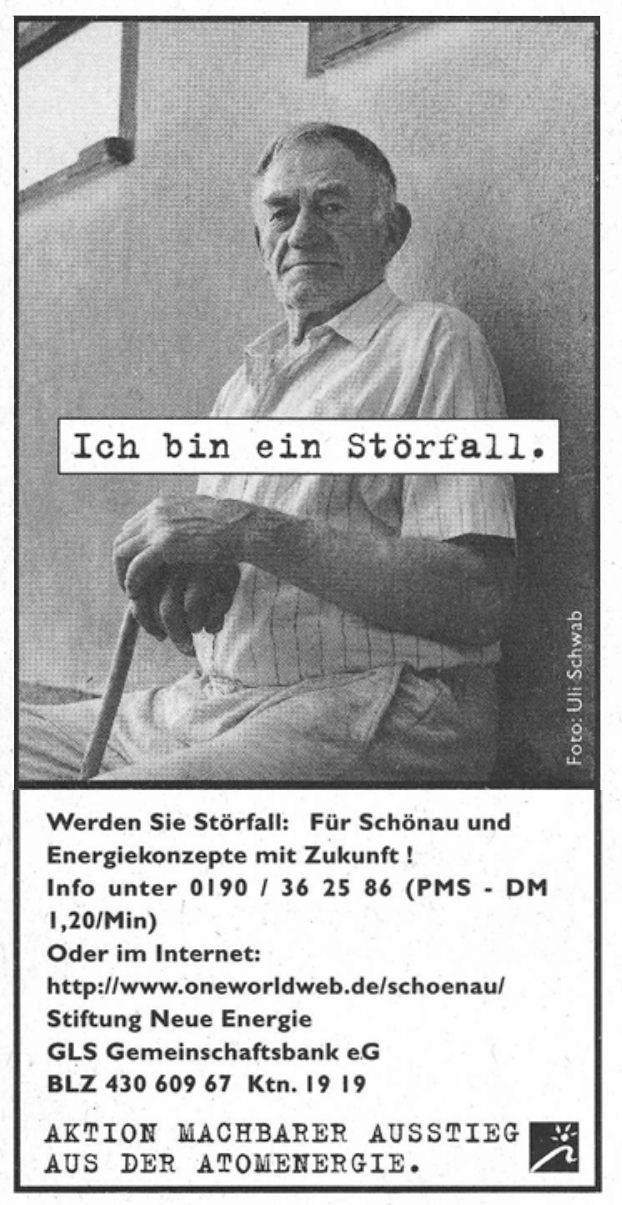

\section{Amoco Oil}

Als eines von mehreren US-amerikanischen Unternehmen, die sich um praktische Ansötze der Umwelikostenrechnung bemühen, hat die Amoco Oil Company für eine ihrer fünf Erdörraffinerien ein Pilotprojekt zur Umweltkostenrechnung durchgeführt (8). Das Projekt beschäftigte sich mit der Berechnung der Umweltkosten am Standort Yorktown, Virginio auf Grundlage des Activity-Based Costing (Prozeßkostenrechnung). Die Vorteile des Activity-Based Costing als Umweltkostenrechnungssystem bestehen darin, daß dieses in das Managementsystem des Unternehmens eingebunden ist, daß Unternehmensziele, Aktivitöten (Prozessen) und Kosten systematisch verknüpft werden und daß das Umweltkostenrechnungssystem stark handlungsbzw. entscheidungsorientiert ist. Mit der Identifizierung von Prozessen im Gemeinkostenbereich und der Verrechnung der anfallenden Gemeinkosten über Prozeßkostensätze wird die Beziehung zwischen den Produkten und den beanspruchten Prozessen berücksichtigt und so eine verursachunggerechtere Zuordnung dieser Kosten zu den Kostenträgern erreicht.

sen Synergieeffekte unberücksichtigt, die sich durch das Zusammenspiel mit anderen Informationssystemen ergeben würden.

Die Umweltkostenrechnung ist im Kontext des betrieblichen Umweltmanagements und auch im Kontext des Controllings zu sehen. Mit Blick auf die Aufgaben und Instrumente des Umweltmanagements (6-7) kommt der Umweltkostenrechnung eher eine flankierende Bedeutung zu. Mit Blick auf den steigenden Kostendruck im internationalen Wettbewerb, kommt umfassenderen Ansätzen der Umweltkostenrechnung wie der Fluß- und Reststoffkostenrechnung eine zentrale Funktion bei der Ermittlung von Kostensenkungspotentialen bei gleichzeitiger Umweltentlastung zu. Würden die in einem Modellprojekt bei der Firma Kunert mittels der Flußkostenrechnung ermittelten Kostensenkungspotentiale auf die gesamte deutsche Wirtschaft übertragen, so ergäbe dies eine Senkung der Gesamtkosten von einem Prozent. Damit ließen sich jährlich Kosten von rund 20 Milliarden Mark einsparen (3).

Festzuhalten gilt, daß Ansätze der Umweltkostenrechnung, die den unternehmenspraktischen sowie den umwelt- und wirtschaftspolitischen Anforderungen genügen sollen, den Informationsbedarf praktischer Entscheidungs- und Nutzungssituation erfassen und das Zusammenspiel mit anderen betrieblichen Informationssystemen sowie die Integration in den Managementprozeß einer kontinuierlichen Verbesserung klar konzipieren müssen.

\section{Anmerkungen}

1) Das Gutachten erscheint im Frühjahr 1997 im Springer Verlag.

2) EPA (ed.): Environmental Accounting Case Studies: Green Accounting at AT\&T. Washington D.C, 1995

3) Kunert AG, Kienbaum Unternehmensberatung GmbH, IMU (Hrsg.): Modellprojekt Umweltkostenmanagement. Abschlufbericht. Immenstadt, 1996

4) EPA (ed.), Environmental Accounting Case Studies: Full Cost Accounting for Decision Making at Ontario Hydro. Washington D.C., 1996

\section{Kunert}

Der Ansatz einer stoff- und energieflußorientierten Kostenrechnung, die auch als Flußkostenrechnung bezeichnet wird, fand 1994/95 in einem Modellprojekt "Umweltkostenmanagement" bei der Firma Kunert die bislang umfassenste Implementierung (2). Die FluBkostenrechnung" hebt sich von bisherigen Ansützen der Umwellkostenrechnung dadurch ab, daß sie keine Unterteilung in "Umweltschutzbedingte" und "nicht-umweltschutzbedingte" Kosten vornimmt. Dazu werden bisherige Kostenbetrachtungen und -zuordnungen auf einzelne Stoff- und Energieflüsse erweitert und "Kostenträger" wie Strom, Gefahrstoffe, Verpackungsmaterialien oder Reststoffe als Kostenbezugseinheit ausgewählt.

Es wird davon ausgegangen, daß mit der Vermeidung bzw. Verminderung von Stoff- und Energieströmen oder deren umweltverträglichere Substitution eine geringere (produktionsbedingte) Umweltbelastung einhergeht. Sie muß sich daher im weiteren nicht mit der Frage beschäftigen, welche betrieblichen Maßnahmen zu welchem Antel dem Umweltschutz dienen. Die Flufkostenrechnung verknüpft systematisch das betriebliche Umwelt- und Stoffstrommanagement mit der Kostenrechnung und ermöglicht eine integrierte Sichtweise ökonomischer und ökologischer Sachverhalte. Auf diesem Weg können außerdem die Kosten des unterlassenen Umweltschutzes erfaßt werden.

\section{5) C. Tuppen (British Telecom) (ed.): Environmental} Accounting in Industry. A practical review. London, 1996 6) K. Fichter: Der Ablauf des Gemeinschaftssystems: mit Öko-Controlling zum zertifizierten Umweltmanagementsystem. in: K. Fichter (Hrsg.): Die EG-Öko-Audit-Verordnung. München/Wien, S. 68

7) BMU/UBA (Hrsg.): Handbuch des Umwelt-Controlling. München, 1995

8) D. Ditz, J. Ranganathan, D. Banks (ed.): Green ledgers: Case Studies in Corporate Environmental Accounting, Baltimore, USA, 1995

\begin{tabular}{|c|}
\hline $\begin{array}{l}\text { Der Autor } \\
\text { Klaus Fichter ist wissenschaftlicher Mitarbeiter am IÖW in } \\
\text { Berlin } \\
\text { Kontakt: IÖW, Giesebrechtstr. } 13,10629 \text { Berlin, Tel. } \\
\text { (030) } 884594-0 \text {, Fax } 8825439\end{array}$ \\
\hline
\end{tabular}


(c) 20I0 Authors; licensee IÖW and oekom verlag. This is an article distributed under the terms of the Creative Commons Attribution Non-Commercial No Derivates License (http://creativecommons.org/licenses/by-nc-nd/3.o/), which permits unrestricted use, distribution, and reproduction in any medium, provided the original work is properly cited. 